\title{
Intrathecal herpes simplex virus type 1 amplicon vector- mediated human proenkephalin reduces chronic constriction injury-induced neuropathic pain in rats
}

\author{
WANGYUAN ZOU, QULIAN GUO, CHAN CHEN, YONG YANG and E. WANG \\ Department of Anesthesiology, Xiangya Hospital, Central South University, Changsha 410008, P.R. China
}

Received November 17, 2010; Accepted January 22, 2011

DOI: $10.3892 / \mathrm{mmr} .2011 .445$

\begin{abstract}
In the present study, we investigated the antinociceptive effect of herpes simplex virus type 1 (HSV-1) amplicon vector-mediated human proenkephalin (hPPE) on chronic constriction injury (CCI)-induced neuropathic pain in rats. Male Sprague-Dawley rats were intrathecally administered normal saline (NS), pHSVIRES-lacZ (SHZ) or recombinant HSV-1 amplicon vector pHSVIRES-hPPE-lacZ (SHPZ), respectively. Once a week for 5 weeks after the intrathecal (i.t.) administration, the expression levels of hPPE mRNA and leu-enkephalin (L-EK) were determined. The paw withdrawal mechanical threshold (PWMT) and paw withdrawal thermal latency (PWTL) were measured before CCI (baseline) on day 3 and once a week for 5 weeks after i.t. administration. The results showed that the PWMT and PWTL in the SHPZ group were significantly increased compared to the thresholds before i.t. administration. The antinociceptive effect of SHPZ reached its peak 3 weeks after i.t. administration and was maintained for 5 weeks. In the rats administered vehicle or SHZ, there were no significant differences between the PWMT or PWTL and the thresholds before i.t. administration. These results indicate that a single i.t. administration of HSV-1 amplicon vector-mediated hPPE attenuated CCI-induced hypersensitivity in rats.
\end{abstract}

\section{Introduction}

Chronic neuropathic pain is a major health problem with few effective therapeutic interventions. At present, treatment with opioids or other methods such as interventional treatments are limited by significant side effects, including gastrointestinal complications, respiratory depression, tolerance and dependence and relative inefficiency (1). These limitations have led

Correspondence to: Dr Qulian Guo, Department of Anesthesiology, Xiangya Hospital, Central South University, 87 Xiangya Road, Changsha 410008, P.R. China

E-mail: qulianguo@yahoo.com.cn

Key words: herpes simplex virus type 1, human enkephalins, neuropathic pain, intrathecal to the search for pain treatments that are more effective and have fewer side effects.

Endogenous opioid peptides play a substantial role in the control of pain perception, and the transport of opioid peptides into the central nervous system (CNS) produces a significant analgesic effect without apparent adverse effects $(2,3)$. Enkephalins, a family of endogenous opioid polypeptides, produce an analgesic effect by acting on the $\delta$ and $\mu$ opioid receptors of the CNS, in particular the $\delta$ opioid receptors. The human proenkephalin (hPPE) gene controls proenkephalin expression, which is converted to bioactive enkephalin (4).

Gene transfer is a novel and useful means for facilitating the expression of target peptides in focal sites within the nervous system, and provides improved efficacy while minimizing potential systemic side effects (5). For the treatment of chronic pain, cell transplantation and non-viral gene therapy approaches are not easily applied for therapeutic purposes due to their low transfection efficiency and the temporary expression of foreign genes (6-8). By contrast, viral vector-mediated transgene expression has the advantages of high transfection efficiency and stable and long-lasting expression of foreign genes $(9,10)$.

Herpes simplex virus type 1 (HSV-1) is particularly well suited for the delivery of genes to neurons (11). Furthermore, HSV-1 amplicon vectors have shown great promise in mediating long-term gene expression in the CNS for the treatment of chronic pain. Over the past several years, a number of investigators have made remarkable progress using HSV-based vectors, mainly via the subcutaneous (s.c.) route, to overexpress inhibitory neurotransmitters in the primary afferents, which attenuate nociceptive neurotransmission at the first synapse between the primary peripheral nociceptor and the second order neuron in the spinal cord (12-14).

The spinal cord is rich in opioid receptors, suggesting that it is a potential transgenic analgesic location. Intrathecal (i.t.) delivery is a commonly used method for chronic pain gene therapy (15-18). hPPE delivered i.t. via the HSV-1 vector may prove to be a novel treatment strategy for chronic neuropathic pain if its expression product produces a stable and prolonged analgesic effect without apparent adverse effects $(19,20)$. Therefore, in this study we investigated the antinociceptive effect of HSV-1 amplicon vector-mediated hPPE, delivered via the i.t route, on chronic constriction injury (CCI)-induced neuropathic pain in rats. 


\section{Methods and materials}

HSV-1 amplicon vector construction and recombination. hPPE gene fragments were cut from pCMVhPPE plasmid and purified. The ligation of the hPPE fragment and HSV-1 amplicon vector pHSVIRES-lacZ (SHZ) was employed to construct the recombinant plasmid pHSVIRES-hPPE-lacZ (SHPZ). HSV-tsk was used as a helper virus for the packaging and amplification of the recombinant plasmid in BHK-21 cells. The amplicon virus titer and the expression of the lacZ gene were examined by $\mathrm{X}$-gal staining. This vector contains the proenkephalin transgene and the lac $Z$ reporter gene. The control vector (SHZ) was identical to SHPZ, except that the inserted cassette contained only the lacZ reporter gene. The final vector was purified at $8 \times 10^{6} \mathrm{pfu} / \mathrm{ml}$.

Animals. Male Sprague-Dawley rats (250-300 g) were purchased from the Shanghai Experimental Animal Center of the Chinese Academy of Sciences. The animals were housed in cages at a controlled temperature of $20 \pm 0.5^{\circ} \mathrm{C}$ and maintained on a $12 \mathrm{~h}$ light-dark cycle with free access to food and water, and were allowed to acclimate for 1 week after arrival. All animal experimental procedures conformed to the guidelines established by the Council of the China Physiologic Society, and were approved by the Administrative Committee of Experimental Animal Care and Use of Central South University. The study adhered to the ethical guidelines of the International Association for the Study of Pain (21).

Chronic constriction injury (CCI). The procedure used to produce the CCI model was first described by Bennett and Xie (22). In brief, each rat was anesthetized by chloral hydrate (300 mg/kg, i.p.). The left common sciatic nerve was exposed at mid-thigh level by blunt dissection through the biceps femoris. Proximal to the sciatic trifurcation, four ligatures (4-0 chromic gut) with $\sim 1-\mathrm{mm}$ spacing were loosely tied around the nerve. The incision was closed in layers. A sham surgery was performed with the sciatic nerve exposed but not ligated. Animals that underwent CCI surgery and exhibited vigorous mechanical and thermal hypersensitivity as a result of the nerve injury were used for the experiments.

Intrathecal catheter implantation. Intrathecal catheters were implanted as described by Yaksh and Rudy (23). In brief, each rat was anesthetized by chloral hydrate $(300 \mathrm{mg} / \mathrm{kg}$, i.p.). The occipital muscles were bluntly separated, and then the cisternal membrane was exposed. Microspinal catheters (inner diameter, $0.12 \mathrm{~mm}$; outer diameter, $0.35 \mathrm{~mm}$ ) were inserted into the subarachnoid space via an incision in the cisterna magna, and advanced $8.0-8.5 \mathrm{~cm}$ caudally to the level of the lumbar enlargement region. Natrium penicillin $(30,000$ units, Huaibei, China) was administered s.c. to prevent infection. Correct intrathecal placement was confirmed by the dragging or paralysis of bilateral hind limbs after the injection of $10 \mu 12 \%$ lidocaine. The rats were allowed to recover for 3 days after implantation and monitored daily for signs of motor deficiency or infection.

Experimental animal groups. Rats were randomly divided into sham (sham), CCI+saline (NS), CCI+pHSVIRES-LacZ
(SHZ) and CCI+pHSVIRES-hPPE-LacZ (SHPZ) groups. The rats in each group were anesthetized by intraperitoneal (i.p) injection of chloral hydrate (300-350 mg/kg), and were then intrathecally administered NS, SHZ or recombinant HSV-1 amplicon vector SHPZ. After the vector $(10 \mu \mathrm{l}$ or $5 \mu \mathrm{l}$ ) was administered, the catheter was flushed with $5 \mu \mathrm{l}$ or $10 \mu \mathrm{l}$ of PBS. The total volume of the vector plus PBS of a single injection was $15 \mu \mathrm{l}$. Once a week for 5 weeks after i.t. administration, the expression of hPPE mRNA and leu-enkephalin (L-EK) in the L4-5 lumbar segments of the spinal cord were determined ( $n=6$ per group). For naloxone reversibility, rats received naloxone $(4 \mathrm{mg} / \mathrm{kg})$ by i.p. injection 3 weeks after i.t. administration (4 weeks after CCI). The paw withdrawal mechanical threshold (PWMT) and paw withdrawal thermal latency (PWTL) were measured before CCI (baseline) on day 3, and once a week for 5 weeks after i.t. administration ( $\mathrm{n}=8$ per group). The PWMT was determined 30 min after i.p. injection of naloxone ( $n=8$ in the $\mathrm{SHZ}$ and SHPZ groups).

Measurement of pain threshold. Pain threshold testing was performed by investigators blinded to the drugs being administered. The PWMT was determined using an automated testing device (Electronic von Frey Anesthesiometer; 2390 series, IITC Life Science, USA) as described previously $(24,25)$. A steel rod (diameter, $0.5 \mathrm{~mm}$ ) was pushed against the ventral surface of the hindpaw with ascending force. The force increased from 0 to $70 \mathrm{~g}$ over a 20 -sec period. When the animal withdrew its hindpaw, the mechanical stimulus was automatically stopped, and the force at which the animal withdrew its paw was recorded to the nearest $0.1 \mathrm{~g}$.

The thermal nociceptive threshold was detected by a Hargreaves apparatus (Plantar test, 7370 series, Ugo Basile, Comerio, Italy) according to a previously described method (26). Rats were placed in clear plastic cages on an elevated glass plate and allowed to acclimate to their surroundings for $30 \mathrm{~min}$ before testing. After acclimation, a constant intensity radiant heat source was focused from underneath the glass aimed at the ventral surface of the hindpaw. A digital timer automatically recorded the duration from the start of the stimuli to paw withdrawal, measured to the nearest $0.1 \mathrm{sec}$. The thermal nociceptive basic threshold of the rats was $\sim 11 \mathrm{sec}$. A cut-off time of $15 \mathrm{sec}$ for irradiation was used to avoid any tissue damage, and $5 \mathrm{~min}$ were allowed between stimulations. Experiments were performed 1 day before and on days $3,7,14,28$ and 35 after surgery.

$R T-P C R$. Rats were anesthetized with ether and sacrificed by decapitation. Total RNA was isolated using Trizol reagent (Life Technologies, Grand Island, NY, USA) according to the manufacturer's protocol. RNA was reversely transcribed into cDNA. Total RNA $(2 \mu \mathrm{g})$ extracted from the spinal cord was reversely transcribed using M-MLV reverse transcriptase (Promega, Madison, WI, USA). The primers (Shanghai Bio-engineering Co.) used in this experiment were 5'-AGAGGCCAATGGAAGTGAGA-3' (sense) and 5'-CAGCTCTTTGGCTTCATCT-3' (antisense) for hPPE, and 5'-CAGCCATGTACGTTGCTATC-3' (sense) and 5'-CAGGTCCAGACGCAGGATGGC-3' (antisense) for $\beta$-actin, which served as internal control. The amplified DNA fragments were 

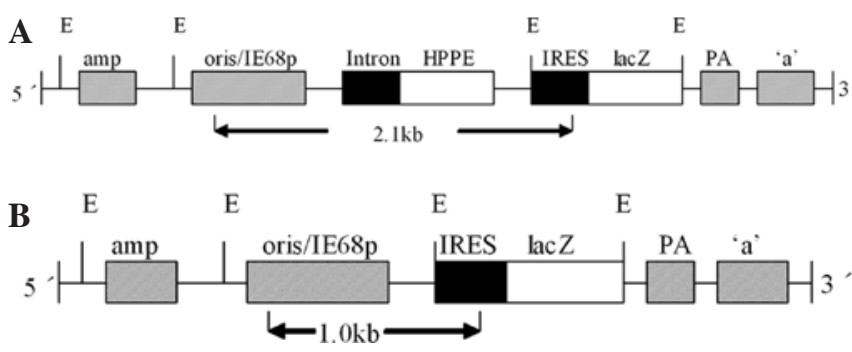

Figure 1. Schematic representation of the pHSVIRES-hPPE-LacZ (SHPZ) and pHSVIRES-LacZ (SHZ) expression vectors. (A) hPPE and lacZ reporter gene were inserted into the recombinant vector SHPZ. (B) The control vector SHZ was identical to SHPZ except that the inserted cassette contained only the lacZ reporter gene.

expected to be $248 \mathrm{bp}$ (hPPE) and $150 \mathrm{bp}$ ( $\beta$-actin). The PCR protocol involved pre-incubation at $94^{\circ} \mathrm{C}$ for $5 \mathrm{~min}$, denaturation at $94^{\circ} \mathrm{C}$ for $45 \mathrm{sec}$, annnealing at $59^{\circ} \mathrm{C}$ for $55 \mathrm{sec}$ and extension at $72^{\circ} \mathrm{C}$ for $60 \mathrm{sec}$ for 38 cycles (hPPE) or 5 cycles ( $\beta$-actin), and a final extension at $72^{\circ} \mathrm{C}$ for $10 \mathrm{~min}$. The amplification products were stored at $4^{\circ} \mathrm{C}$ and electrophoresed on $1.8 \%$ agarose gel. The integrated optical density (IOD) ratio of hPPE to $\beta$-actin represented the relative expression level of the hPPE.

Detection of leu-enkephalin in the spinal cord. Leu-enkephalin (L-EK) in the spinal cord was assayed using a L-EK radio-125 immunoassay (IRIA) kit (Department of Neurobiology, Second Military Medical University, China). At 1, 2, 3, 4 and 5 weeks after i.t. administration, rats in each group were sacrificed by decapitation. L4-5 lumbar segments of the spinal cord $(50 \mathrm{mg}$ ) were added to $500 \mu \mathrm{TE}$ buffer. The supernatant was separated from the homogenate of the spinal cord by centrifugation, and the supernatant was frozen at $-70^{\circ} \mathrm{C}$ until analysis. The total reaction volume was $300 \mu$, including the sample of analyte organizations supernatant $(100 \mu \mathrm{l})$, L-EK antiserum $(100 \mu \mathrm{l})$ and ${ }^{125} \mathrm{I}-\mathrm{L}-\mathrm{EK}(100 \mu \mathrm{l})$. The L-EK content in the supernatant was assayed by the serial saturated method according to the manufacturer's protocol. Protein content was measured by a previously described method (27). The secretion level was standardized and expressed in $\mathrm{pg} / \mathrm{ml}$ protein.

Statistical analysis. Data are expressed as the means \pm SEM. Two-way repeated-measures analysis of variance (ANOVA) was used to detect differences in hPPE mRNA, L-EK and pain thresholds among groups. P-values $<0.05$ were considered statistically significant.

\section{Results}

hPPE expression detected after intrathecal delivery of HSV-I vector-mediated gene transfer. Schematic representations of the pHSVIRES-hPPE-LacZ and pHSVIRES-LacZ expression vectors are shown in Fig. 1. As shown in Fig. $2 \mathrm{~A}$ and $\mathrm{B}$, the 150 -bp product of $\beta$-actin as an internal control was detected in the NS, SHZ and SHPZ groups. hPPE mRNA expression was detected in the SHPZ group only, indicating that the hPPE gene was successully transfected into the spinal cord 1-5 weeks after i.t. administration of SHPZ. hPPE mRNA expression reached its peak 3 weeks after transfection.
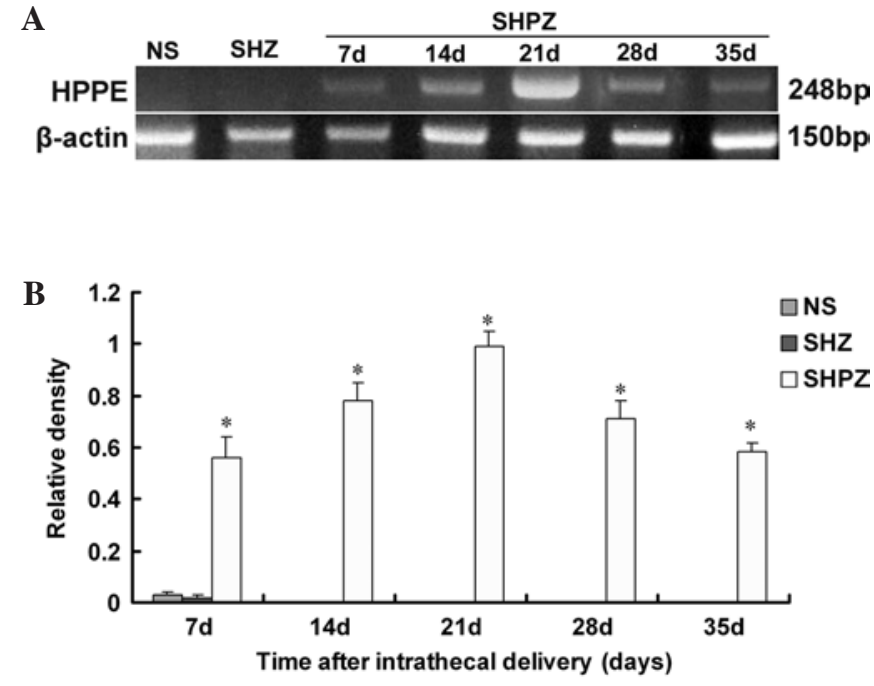

Figure 2. (A) Expression of hPPE mRNA in L4-5 lumbar segments of the spinal cord (248 bp). (B) Relative hPPE mRNA expression (determined by RT-PCR) in the spinal dorsal horn 1-5 weeks after i.t. delivery. Data are presented as the means \pm SEM ( $n=6$ per group).

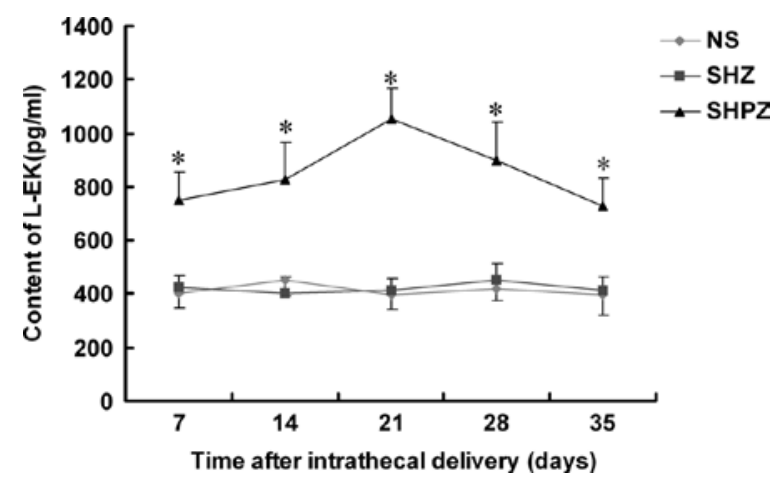

Figure 3. L-EK content in the sham, saline, SHZ and SHPZ groups 1-5 weeks after i.t. injection. ${ }^{*} \mathrm{P}<0.05$ vs. sham and $\mathrm{SHZ}$ groups ( $\mathrm{n}=6$ per group). There was an increase in L-EK levels from day 7 to day 35 in the SHPZ-treated CCI rats, peaking on day 21 ( $n=6$ per group).

Time course of leu-enkephalin expression after hPPE gene transfer. L-EK levels in the spinal cord of the rats were lower in the NS and SHZ groups than in the SHPZ group after i.t. administration $(\mathrm{P}<0.05)$. There was an increase in L-EK from day 7 to day 35 in SHPZ-treated CCI rats, peaking on day 21 ( $n=6$ per group) (Fig. 3 ).

Intrathecal delivery of SHPZ significantly attenuated allodynia and thermal hyperalagesia. CCI, but not sham surgery, produced significant mechanica allodynia and thermal hyperalgesia $(\mathrm{P}<0.05)$. The time course of the PWMT and PWTL is presented in Fig. 4. Intrathecal administration of SHPZ, but not SHZ, attenuated CCI-induced PWMT and PWTL as of day 7, reaching a peak on day 21 after the procedure $(\mathrm{P}<0.05$ vs. the NS and SHZ groups, and $\mathrm{P}<0.01$ vs. the rats pre-i.t. administration). Thereafter, the PWMT and PWTL in the SHPZ group tended to decrease, but remained elevated throughout the observation period of 35 days $(\mathrm{P}<0.05$ on days $14,21,28$ and 35 vs. the NS and SHZ groups, and $\mathrm{P}<0.01$ vs. the rats pre-i.t. 
A

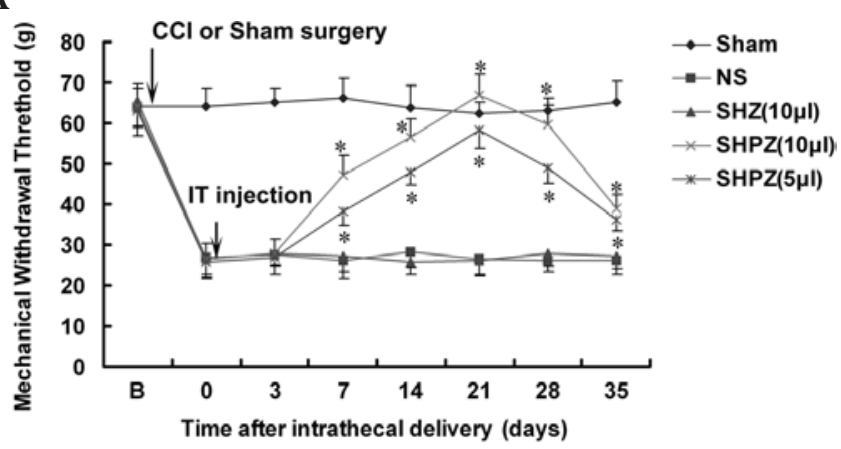

B

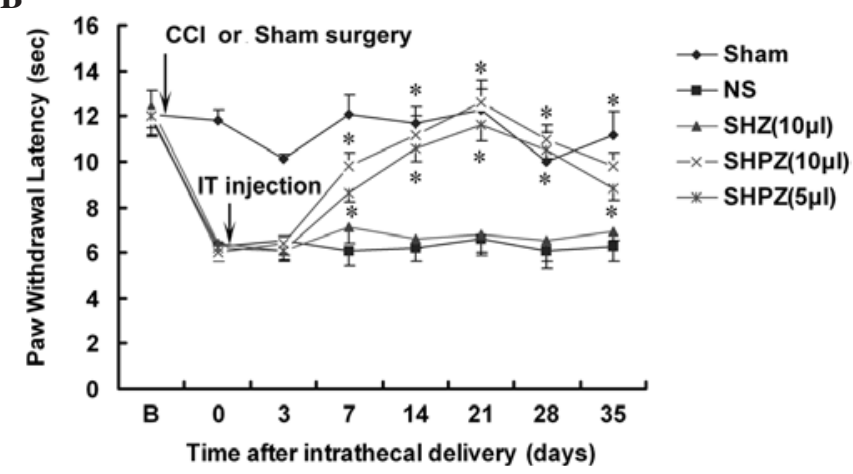

Figure 4. Changes in (A) tactile allodynia (PWMT) and (B) thermal hyperalgesia (PWTL) after CCI and intrathecal (i.t.) delivery of SHPZ. Rats were left unoperated in the sham group (sham). The other three groups of rats underwent CCI. After 1 week, the rats were administered vehicle (saline), pHSVIRES-LacZ (SHZ) or pHSVIRES-hPPE-LacZ (SHPZ) (5 or $10 \mu 1$ in a total volume of $15 \mu \mathrm{l})$. The PWMT was measured weekly for 5 weeks after i.t. delivery. B, baseline pain threshold of rats before CCI; 0, time point before i.p. delivery; 3 , time point 3 days after i.p. delivery/10 days after CCI. Data are presented as the means \pm SEM. Significance was defined as ${ }^{*} \mathrm{P}<0.05$ vs. the NS or SHZ groups ( $\mathrm{n}=8$ per group).

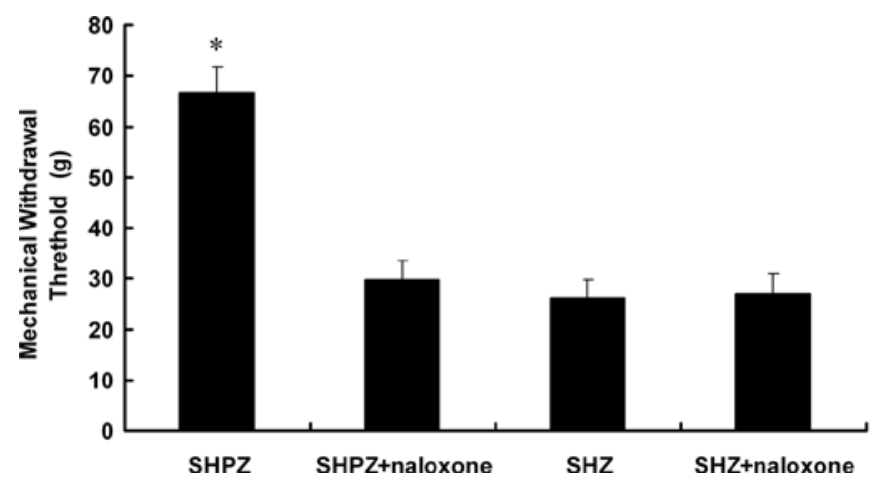

Figure 5. Intraperitoneal injection of naloxone $(4 \mathrm{mg} / \mathrm{kg})$ reversed the antiallodynic effect of SHPZ in animals tested 3 weeks after vector administration (4 weeks after CCI). The PWMT was determined 30 min after injection of naloxone, and the reversal time of antiallodynic effect was maintained for almost 8 h. Significance was defined as ${ }^{*} \mathrm{P}<0.05$ vs. SHPZ plus naloxonetreated rats ( $n=6$ per group).

administration; $n=8$ per group). The differences between the PWMT and PWTL of the SHPZ group in comparison with the SHZ group and with the sham-operated group were significally different $(\mathrm{P}<0.05)$. The high-dose SHPZ $(10 \mu \mathrm{l})$ produced a stronger antinociceptive effect than the low-dose SHPZ (5 $\mu \mathrm{l})$, indicating that the antinociceptive effect of intrathecal delivery of SHPZ was dose-dependent (Fig. 4A and B).
In order to confirm that these pain-related behavior changes were caused by endogenous opioid peptides in the spinal cord, rats in the SHZ and SHPZ groups ( $n=6$ per group) received i.p. administration of naloxane $(4 \mathrm{mg} / \mathrm{kg})$, an opioid receptor antagonist. As shown in Fig. 5, 3 weeks after i.t. administration of SHZ or SHPZ, naloxone reversed the antiallodynic effect (indicated by a reduction in PWMT) in the animals receiving SHPZ, while no significant effects were observed in the SHZ group.

\section{Discussion}

Neuropathic pain is a complex chronic pain state that is usually accompanied by damage to or dysfunction of the peripheral or central nervous system. This type of pain is often insensitive to traditional pain drugs or surgical intervention. Therefore, this study evaluated a different treatment strategy: in vivo targeted gene transfer via i.t. delivery to the spinal cord. The results indicate that HSV-1 vector-mediated hPPE gene transfer via the i.t. route attenuated neuropathic pain in the rats. hPPE expression was observed in the spinal cord 1-5 weeks after i.t. administration of SHPZ. Levels of the hPPE-derived protein product L-EK in the spinal cord increased markedly after the administration of SHPZ, confirming the gene transcription and protein translation. Molecular and biochemical changes in the animals receiving the hPPE gene transfection resulted in the elevation of the pain threshold as measured by the PWMT and PWTL. This was reversed by naloxone, suggesting that the pain-related behavior changes were mediated by the opioid receptor in the spinal cord.

In the present study, the human proenkephalin gene (hPPE) was translated and degraded into two types of short peptides with five amino acids through transcription into host cells: M-enkephalin and L-EK $(28,29)$, both of which have a potent analgesic effect in vivo. L-EK is one of the endogenous opioid peptides that have an analgesic effect on the CNS through the $\delta$ and $\mu$ opioid receptors, particularly the $\delta$-opioid receptors. When high doses of enkephalin $(10 \mathrm{mg})$ are administered to rats intrathecally or introduced into the CNS, the opioid peptides produce a significant analgesic effect without adverse reactions, such as marked respiratory and circulatory dysfunction; thus, the safety coefficient of enkephalin is higher than that of morphine (30). Based on these and our findings, hPPE may be an ideal target gene for chronic pain gene therapy.

The natural ability of replication-defective HSV vectors to transduce peripheral neurons by s.c. injection provides a platform for the investigation of therapeutic transgene delivery. Previous studies have found that HSV-mediated hPPE transfer through s.c. injection produces analgesic effects against inflammatory, neuropathic and bone cancer pain in animal models (12-14). However, the best route(s) for effective gene transfer remain to be determined.

Pain modulating genes such as interleukin-2 have been intrathecally transfected using adenoviral vectors (31). Intrathecal delivery of recombinant adeno-associated virus expressing the anti-inflammatory gene product human IL-10 or brain-derived neurotrophic factor has been shown to attenuate chronic neuropathic pain after partial nerve injury (32-34). These studies indicate that the i.t. route for gene delivery to the spinal cord may be another viable option for gene therapy of chronic pain. 
This study provides evidence that the i.t. delivery of HSV-1 vector-mediated SHPZ reduced neuropathic pain induced by $\mathrm{CCI}$ in rats. However, there were no differences in the thermal hyperalgesia and tactile allodynia thresholds on day 3 in the SHPZ group compared to the control groups, suggesting that the HSV-1 vector-mediated hPPE gene may not be suitable for the treatment of acute pain. The differences in the pain threshold were significant at week 1 and reach an analgesic peak at week 3 , and were maintained for 5 weeks after i.t. SHPZ administration. This suggests that the therapeutic proteins were synthesized and exerted their biological effect in the spinal cord continuously, and might therefore provide an analgesic effect for the treatment of chronic pain. Furthermore, i.t. delivery of SHPZ attenuated pain thresholds in a dose-dependent manner.

Zhao et al (35) reported that i.t. morphine administration is likely to be more effective than s.c. injection in the treatment of neuropathic pain. Since the spinal cord is rich in opioid receptors, i.t. administration of SHPZ may provide a shorter time to onset and longer duration of the analgesic effect compared to s.c. injection. However, when comparing gene transfer by i.t. delivery, as in this study, and s.c. injection, conducted in previous studies, there were no apparent differences between the time to onset and the duration of the analgesic effect (12-14). It remains to be determined whether this finding is related to the final concentration of the purified vectors used, or other factors.

In conclusion, the present study demonstrated that single intrathecal administration of HSV-1 amplicon vector-mediated human proenkephalin attenuated CCI-induced hypersensitivity in rats.

\section{Acknowledgements}

This study was supported by the National Science Council of China (grant nos. 30801074 and 30772080). The authors thank Dr Jian-Zhong Sun of the Department of Anesthesiology, Jefferson Medical College, Thomas Jefferson University, for the critical reading of the manuscript.

\section{References}

1. Przewlocki R and Przewlocka B: Opioids in neuropathic pain Curr Pharm Des 11: 3013-3025, 2005.

2. Egleton RD, Abbruscato TJ, Thomas SA and Davis TP: Transport of opioid peptides into the central nervous system. J Pharm Sci 87: 1433-1439, 1998.

3. Takemori AE and Portoghese PS: Enkephalin antinociception in mice is mediated by delta 1 - and delta 2-opioid receptors in the brain and spinal cord, respectively. Eur J Pharmacol 242: $145-150,1993$

4. Liu F, Housley PR and Wilson SP: Initial processing of human proenkephalin in bovine chromaffin cells. J Neurochem 67 $1457-1462,1996$

5. Wolfe D, Hao S, Hu J, et al: Engineering an endomorphin-2 gene for use in neuropathic pain therapy. Pain 133: 29-38, 2007.

6. Fink D, Mata M and Glorioso JC: Cell and gene therapy in the treatment of pain. Adv Drug Deliv Rev 55: 1055-1064, 2003.

7. Springer SL, Vite CH, Polesky AC, Kesari S, Fraser NW and Wolfe JH: Infection and establishment of latency in the dog brain after direct inoculation of a nonpathogenic strain of herpes simplex virus-1. J Neurovirol 7: 149-154, 2001.

8. Wilson SP and Yeomans DC: Virally mediated delivery of enkephalin and other neuropeptide transgenes in experimental pain models. Ann NY Acad Sci 971: 515-521, 2002.

9. Fink DJ and Mata M: HSV gene transfer in the treatment of chronic pain. Sheng Li Xue Bao 60: 610-616, 2008.

10. Metharom P, Takyar S, Xia HH, et al: Novel bovine lentiviral vectors based on Jembrana disease virus. J Gene Med 2: 176-185, 2000.
11. Glorioso JC and Fink DJ: Herpes vector-mediated gene transfer in treatment of diseases of the nervous system. Annu Rev Microbiol 58: 253-271, 2004

12. Hao S, Wolfe D, Glorioso JC, Mata M and Fink DJ: Effects of transgene-mediated endomorphin-2 in inflammatory pain. Eur J Pain 13: 380-386, 2009.

13. Meunier A, Latremoliere A, Mauborgne A, et al: Attenuation of pain-related behavior in a rat model of trigeminal neuropathic pain by viral-driven enkephalin overproduction in trigeminal ganglion neurons. Mol Ther 11: 608-616, 2005.

14. Goss JR, Harley CF, Mata M, et al: Herpes vector-mediated expression of proenkephalin reduces bone cancer pain. Ann Neurol 52: 662-665, 2002.

15. Hao S, Mata M, Glorioso JC and Fink DJ: Gene transfer to interfere with TNFalpha signaling in neuropathic pain. Gene Ther 14: 1010-1016, 2007.

16. Beutler AS, Banck MS, Walsh CE and Milligan ED: Intrathecal gene transfer by adeno-associated virus for pain. Curr Opin Mol Ther 7: 431-439, 2005.

17. Gabra BH, Kessler FK, Ritter JK, Dewey WL and Smith FL: Decrease in N-methyl-D-aspartic acid receptor-NR2B subunit levels by intrathecal short-hairpin RNA blocks group I metabotropic glutamate receptor-mediated hyperalgesia. J Pharmacol Exp Ther 322: 186-194, 2007.

18. Ledeboer A,Jekich BM,Sloane EM, et al: Intrathecal interleukin-10 gene therapy attenuates paclitaxel-induced mechanical allodynia and proinflammatory cytokine expression in dorsal root ganglia in rats. Brain Behav Immun 21: 686-698, 2007.

19. Wu HE, Hung KC, Mizoguchi H, Nagase H and Tseng LF: Roles of endogenous opioid peptides in modulation of nocifensive response to formalin. J Pharmacol Exp Ther 300: 647-654, 2002.

20. Kiguchi N, Maeda T, Tsuruga M, et al: Involvement of spinal Met-enkephalin in nicotine-induced antinociception in mice. Brain Res 1189: 70-77, 2008.

21. Zimmermann M: Ethical guidelines for investigations of experimental pain in conscious animals. Pain 16: 109-110, 1983.

22. Bennett GJ and Xie YK: A peripheral mononeuropathy in rat that produces disorders of pain sensation like those seen in man. Pain 33: 87-107, 1988.

23. Yaksh TL and Rudy TA: Chronic catheterization of the spinal subarachnoid space. Physiol Behav 17: 1031-1036, 1976.

24. Chaplan SR, Bach FW, Pogrel JW, Chung JM and Yaksh TL: Quantitative assessment of tactile allodynia in the rat paw. J Neurosci Methods 53: 55-63, 1994.

25. Villetti G, Bergamaschi M, Bassani F, et al: Antinociceptive activity of the N-methyl-D-aspartate receptor antagonist $\mathrm{N}-(2-$ Indanyl)-glycinamide hydrochloride (CHF3381) in experimental models of inflammatory and neuropathic pain. J Pharmacol Exp Ther 306: 804-814, 2003.

26. Hargreaves K, Dubner R, Brown F, Flores C and Joris J: A new and sensitive method for measuring thermal nociception in cutaneous hyperalgesia. Pain 32: 77-88, 1988.

27. Lowry OH, Rosebrough NJ, Farr AL and Randall RJ: Protein measurement with the Folin phenol reagent. J Biol Chem 193: 265-275, 1951.

28. Noda M, Furutani Y, Takahashi $\mathrm{H}$, et al: Cloning and sequence analysis of cDNA for bovine adrenal preproenkephalin. Nature 295: 202-206, 1982.

29. LaForge KS, Nyberg F and Kreek MJ: Primary structure of guinea pig preprodynorphin and preproenkephalin mRNAs: multiple transcription initiation sites for preprodynorphin. Brain Res Bull 63: 119-126, 2004

30. Atchison SR, Durant PA and Yaksh TL: Cardiorespiratory effects and kinetics of intrathecally injected D-Ala2-D-Leu5-enkephalin and morphine in unanesthetized dogs. Anesthesiology 65: 609-616, 1986.

31. Yao MZ, Gu JF, Wang JH, et al: Interleukin-2 gene therapy of chronic neuropathic pain. Neuroscience 112: 409-416, 2002.

32. Milligan ED, Sloane EM, Langer SJ, et al: Repeated intrathecal injections of plasmid DNA encoding interleukin-10 produce prolonged reversal of neuropathic pain. Pain 126: 294-308, 2006.

33. Milligan ED, Soderquist RG, Malone SM, et al: Intrathecal polymer-based interleukin-10 gene delivery for neuropathic pain. Neuron Glia Biol 2: 293-308, 2006.

34. Eaton MJ, Blits B, Ruitenberg MJ, Verhaagen J and Oudega M: Amelioration of chronic neuropathic pain after partial nerve injury by adeno-associated viral (AAV) vector-mediated over-expression of BDNF in the rat spinal cord. Gene Ther 9: 1387-1395, 2002.

35. Zhao C, Tall JM, Meyer RA and Raja SN: Antiallodynic effects of systemic and intrathecal morphine in the spared nerve injury model of neuropathic pain in rats. Anesthesiology 100: 905-911, 2004. 